\title{
Pharmacokinetics of Alternative Administration Routes of Melatonin: A Systematic Review
}

\author{
Authors \\ Affiliation \\ Key words
bioavailability
intranasal
transdermal
transmucosal
subcutaneous
}

\section{Zetner, L. P. H. Andersen, J. Rosenberg}

Department of Surgery, Center for Perioperative Optimization, Herlev Hospital, University of Copenhagen, Herlev, Denmark received 15.09 .2015

accepted 06.10.2015

Bibliography

DOI http://dx.doi.org/

10.1055/s-0035-1565083

Published online:

October 29, 2015

Drug Res 2016;

66: 169-173

(c) Georg Thieme Verlag KG

Stuttgart · New York

ISSN 2194-9379

Correspondence

\section{Zetner}

Department of Surgery

Center for Perioperative

Optimization

Herlev Hospital

Herlev Ringvej 75

2730 Herlev

Denmark

Tel.: + 45/27/29 1376

Fax: +45/38/68 4009

dennis.zetner@gmail.com

\section{Abstract}

$\nabla$

Background: Melatonin is traditionally administered orally but has a poor and variable bioavailability. This study aims to present an overview of studies investigating the pharmacokinetics of alternative administration routes of melatonin.

Methods: A systematic literature search was performed and included experimental or clinical studies, investigating pharmacokinetics of alternative administration routes of melatonin in vivo. Alternative administration routes were defined as all administration routes except oral and intravenous.

Results: 10 studies were included in the review. Intranasal administration exhibited a quick absorption rate and high bioavailability. Transdermal administration displayed a vari-

\section{Introduction}

$\nabla$

Melatonin is a hormone produced by the pineal gland, regulating the circadian rhythm in mammals [1]. Exogenous administration of melatonin improves sleep quality [2], reduces jet lag [3], and possesses analgesic [4,5], anti-oxidative [6,7] and anti-inflammatory effects [6]. Melatonin is traditionally administered orally, but the drug displays a poor and variable bioavailability due to an extensive first pass metabolism [8-11]. In order to optimize tissue delivery of melatonin, alternative administration routes, other than oral and intravenous formulations, may be required. The aim of this study was to present an overview of studies investigating the pharmacokinetics of alternative administration routes of melatonin in animals and in humans. able absorption rate and possible deposition of melatonin in the skin. Oral transmucosal administration of melatonin exhibited a high plasma concentration compared to oral administration. Subcutaneous injection of melatonin displayed a rapid absorption rate compared to oral administration.

Conclusion: Intranasal administration of melatonin has a large potential, and more research in humans is warranted. Transdermal application of melatonin has a possible use in a local application, due to slow absorption and deposition in the skin. Oral transmucosal administration may potentially be a clinically relevant due to avoiding first-pass metabolism. Subcutaneous injection of melatonin did not document any advantages compared to other administration routes.

\section{Materials and Methods}

This systematic review was conducted in accordance to the PRISMA and PRISMA-P guidelines $[12,13]$ (PROSPERO register, registration number: CRD42015017042). The literature search was performed on February $25^{\text {th }} 2015$ in PubMed and Embase databases. The review included experimental or clinical studies written in English, investigating pharmacokinetics of alternative administration routes of melatonin in vivo. Alternative administration routes were defined as all administration routes except oral and intravenous. Studies were identified using the search terms ((((pharmacokinetic) OR pharmacokinetics) OR bioavailability)) AND melatonin) AND (((human) OR humans) OR animal) OR animals). The "all fields" setting was applied for all search terms. Furthermore, a manual "snowball" search was performed in the reference lists of the studies included. 
2 authors (DZ, LPHA) individually assessed records found in the primary literature search. Disagreements were resolved by discussion. Full-text studies were obtained, evaluated, and included on the basis of the inclusion criteria. Study design, number of subjects, administration route, control/comparison and pharmacokinetic outcomes were evaluated for each study.

The pharmacokinetic outcomes studied in this review were; dose, peak plasma concentration $\left(\mathrm{C}_{\max }\right)$, time to maximal concentration $\left(\mathrm{T}_{\max }\right)$, volume of distribution $\left(\mathrm{V}_{\mathrm{D}}\right)$, elimination halflife $\left(T_{1 / 2}\right)$, clearance $(C L)$ and absolute bioavailability. Only studies presenting one or more of these outcomes calculated from plasma concentrations were included. Data from each study are referred with no transformation or interpretation. The results presented in the review are median or mean values as reported in the studies, without safety intervals.

\section{Results}

The primary search identified 990 records ( 0 Fig. 1 ), whereof 128 duplicates were removed. We screened 862 records on title and abstract. A total of 36 full-text studies were assessed for eligibility, of which 10 studies were included in the final review. The included studies administered melatonin intranasally (I.N.) [14-17], transdermally [18-21], oral transmucosally [20,22] and subcutaneously [23] ( $\odot$ Table 1 ).

\section{Intranasal administration}

In humans, a cross-over study in 3 volunteers administered either $0.4 \mathrm{mg}$ melatonin I.N. or $0.2 \mathrm{mg}$ intravenously (I.V.) on 2 separate study days [15]. The study reported a $\mathrm{T}_{\max }$ of $5 \mathrm{~min}$ for I.N. administration and $10 \mathrm{~min}$ for I.V. administration. No other outcomes for plasma values were reported.

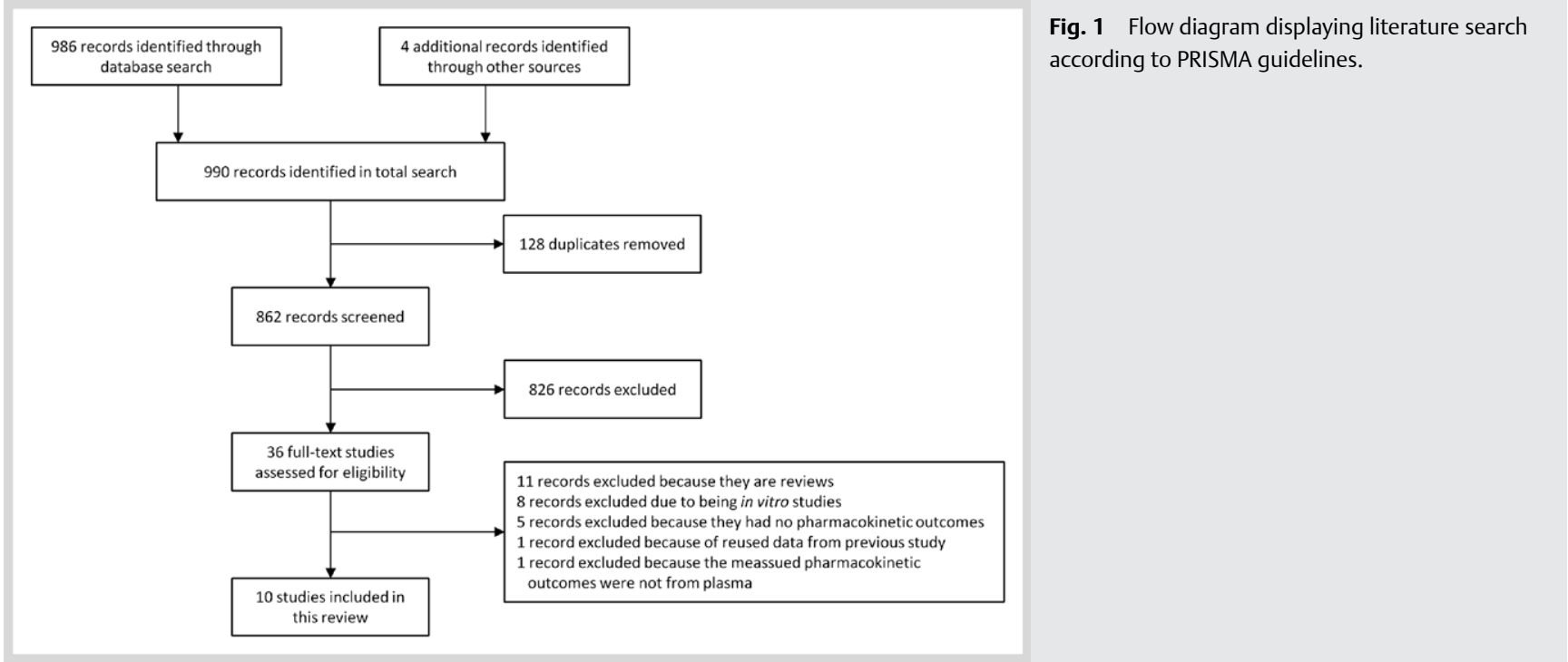

Table 1 Overview of included studies.

\begin{tabular}{|c|c|c|c|c|c|c|}
\hline Study reference & Study design & Species (n) & Dose of melatonin & Administration route & Comparison & Outcomes \\
\hline [14] & Crossover & Human (3) & $0.4 \mathrm{mg}$ & Intranasal & I.V. melatonin & $\mathrm{T}_{\max }$ \\
\hline [15] & Case-control & Rabbit (6) & $1.5 \mathrm{mg}$ & Intranasal & $\begin{array}{l}\text { I.V. melatonin } \\
\text { I.N. melatonin + GC }\end{array}$ & $\begin{array}{l}T_{\max } \\
T_{1 / 2} \\
\text { Bioavailability }\end{array}$ \\
\hline [16] & Case-control & Rabbit (12) & $0.1 \mathrm{mg} / \mathrm{kg}$ & Intranasal & $\begin{array}{l}\text { I.V. melatonin } \\
\text { I.N. SMS w/melatonin }\end{array}$ & $\begin{array}{l}T_{\max } \\
T_{1 / 2} \\
\text { Bioavailability }\end{array}$ \\
\hline [13] & Crossover & Rat (8) & $0.04 \mathrm{mg}$ & Intranasal & I.V. melatonin & $\mathrm{T}_{\max }$ \\
\hline [17] & Crossover & Human (8) & $2.1 \mathrm{mg}$ & Transdermal & Placebo & $\mathrm{T}_{\max }$ \\
\hline [18] & Crossover & Human (2) & $3.6 \mathrm{mg}$ & Transdermal & Baseline & $\begin{array}{l}\mathrm{T}_{\max } \\
\mathrm{T}_{1 / 2} \\
\mathrm{~T}_{1 / 2 \alpha}\end{array}$ \\
\hline [19] & Crossover & Human (12) & $\begin{array}{l}0.5 \mathrm{mg} \\
8 \mathrm{mg}\end{array}$ & $\begin{array}{l}\text { Transmucosal } \\
\text { Transdermal }\end{array}$ & OCR melatonin & $\mathrm{T}_{\max }$ \\
\hline [20] & Crossover & Human (6) & $\begin{array}{l}20 \mathrm{mg} \\
100 \mathrm{mg}\end{array}$ & Transdermal & Baseline & $\mathrm{T}_{\max }$ \\
\hline [21] & Crossover & Human (8) & $5 \mathrm{mg}$ & Sublingual spray & Oral melatonin & $\begin{array}{l}T_{\max } \\
T_{1 / 2}\end{array}$ \\
\hline [22] & Crossover & Sheep (2) & $\begin{array}{l}1 \mathrm{mg} \\
0.1 \mathrm{mg}\end{array}$ & $\begin{array}{l}\text { Subcutaneous injec- } \\
\text { tion }\end{array}$ & Oral melatonin & $\begin{array}{l}T_{\max } \\
T_{1 / 2}\end{array}$ \\
\hline
\end{tabular}

I.N. SMS: intranasal starch microspheres, + GC: with sodium glycocholate, OCR: oral controlled release 
A cross-over study in 6 rabbits investigated bioavailability of intranasal administration of melatonin [16]. The study administered I.V. melatonin, I.N. melatonin and I.N. melatonin with sodium glycocholate. In all 3 administrations, the administered dose was $1.5 \mathrm{mg}$. The study documented similar $\mathrm{T}_{\max }$ values of $5 \mathrm{~min}$ in all 3 administrations routes (first measurement). Mean $\mathrm{T}_{1 / 2}$ for melatonin was $13 \mathrm{~min}$ for I.V. administration and $14 \mathrm{~min}$ for both I.N. and I.N. + GC administration. A mean bioavailability of 55 and $94 \%$ was documented for I.N. melatonin and I.N. melatonin with sodium glycocholate, respectively.

A cross-over study including 8 rats studied I.N. administration of melatonin [14]. The study administered $0.04 \mathrm{mg}$ of melatonin I.V. and I.N. $\mathrm{T}_{\max }$ was documented as $2.5 \mathrm{~min}$ in both I.V. and I.N. administration (first measurement).

A case-control study investigated I.N. administration of melatonin in 12 rabbits [17]. Melatonin was administered I.V., I.N. or I.N. including starch microspheres in doses of $0.1 \mathrm{mg} / \mathrm{kg}$. $\mathrm{T}_{\max }$ values of 4.70 and $7.80 \mathrm{~min}$ were documented for I.N. melatonin and I.N. with starch microspheres with melatonin, respectively. $\mathrm{T}_{1 / 2}$ values were $5.6 \mathrm{~min}$ and $12.3 \mathrm{~min}$ in I.N. melatonin and I.N. with starch microspheres with melatonin, respectively. The bioavailability was $69.72 \%$ for I.N. administration and $84.07 \%$ for I.N. with starch microspheres.

\section{Transdermal administration}

Transdermal application of melatonin was examined in 8 humans in a randomized, double-blinded, cross-over placebocontrolled trial [18]. Patches containing $2.1 \mathrm{mg}$ melatonin, or a placebo, were applied and followed by a wash-out period of 7-16 days. A mean $\mathrm{T}_{\max }$ of $8.58 \mathrm{~h}$ was documented.

A cross-over study in 2 humans investigated transdermal delivery of a nanoparticle gel containing melatonin [19]. The gel, containing $3.6 \mathrm{mg}$ melatonin was applied to a $9 \mathrm{~cm}^{2}$ skin area. $\mathrm{T}_{\max }$ was 12.99 and $18.12 \mathrm{~h}$. $\mathrm{T}_{1 / 2}$ was 5.02 and $10.02 \mathrm{~h}$.

A cross-over study compared transdermal, transmucosal and oral controlled-release administration of $8 \mathrm{mg}$ of melatonin in 12 humans [20]. The study demonstrated a $\mathrm{T}_{\max }$ of $13 \mathrm{~h}$ for the transdermal patches. Patches were removed after $10 \mathrm{~h}$, and the plasma concentration of melatonin continued to rise, suggesting a deposition of melatonin in the skin.

Another study examined transdermal application of melatonin in 2 alcoholic solutions in 6 humans [21]. 3 subjects received $20 \mathrm{mg}$ of melatonin ( $1 \%$ melatonin in a $70 \%$ alcohol solution), whereas 3 received $100 \mathrm{mg}$ of melatonin (5\% in a $70 \%$ alcoholic solution). The solutions were applied to the scalp of the subjects. The 2 subject groups demonstrated the following $\mathrm{T}_{\text {max }}$ : For the $20 \mathrm{mg}$ melatonin group it was 2,8 and $8 \mathrm{~h}$, while the $100 \mathrm{mg}$ group was 1,1 and $6 \mathrm{~h}$, respectively.

\section{Oral transmucosal administration}

A cross-over study in humans compared transdermal, transmucosal and oral controlled-release administration of melatonin [20]. They applied a $0.5 \mathrm{~cm}^{2}$ mucoadhesive buccal patch containing $0.5 \mathrm{mg}$ melatonin in 12 subjects. They documented a $\mathrm{T}_{\max }$ of $474 \mathrm{~min}$ for the buccal patches. Also, $0.23 \mathrm{mg}$ of melatonin was left in the patch after removal, indicating that $0.27 \mathrm{mg}$ of the melatonin had been absorbed.

An open-label, randomized cross-over study in 8 humans compared a sublingual melatonin spray with oral tablets, both containing $5 \mathrm{mg}$ of melatonin [22]. The sublingual administration displayed a mean $\mathrm{T}_{\max }$ of $42.5 \mathrm{~min}$. The study found a signifi- cantly higher $\mathrm{C}_{\max }$ for the spray at $17.2 \mathrm{ng} / \mathrm{mL}$ compared to oral tablets at $12.4 \mathrm{ng} / \mathrm{mL}$. The mean $\mathrm{T}_{1 / 2}$ was $54.0 \mathrm{~min}$.

\section{Subcutaneous injection}

A cross-over study in 2 sheep investigated subcutaneous injection compared to oral administration of melatonin [23]. The sheep were injected with either $1 \mathrm{mg}$ of melatonin in a saline solution, or $0.1 \mathrm{mg}$ melatonin diluted in peanut oil. Furthermore, oral melatonin was administered after a wash-out period of 2 days. The study demonstrated a $\mathrm{T}_{\max }$ of $15 \mathrm{~min}$ and $\mathrm{T}_{1 / 2}$ of $30 \mathrm{~min}$ in both subcutaneous formulations, and $\mathrm{T}_{\max }$ of $30 \mathrm{~min}$ for the oral melatonin.

\section{Discussion \\ $\nabla$}

This systematic review demonstrated that quite limited data on the alternative administration routes of melatonin existed. Studies investigated pharmacokinetic variables following I.N., transdermal, buccal, sublingual and subcutaneous administration. The studies varied extensively in investigated subject, pharmacokinetic outcomes, investigational periods, melatonin doses, and formulations. Our review documented that intranasal administration may have clinical relevance, in circumstances where a systemic effect is wanted, due to rapid $\mathrm{T}_{\max }$ and high bioavailability.

Exogenous melatonin improves sleep quality and reduces jetlag. Furthermore, recent studies in surgical patients documented analgesic, anti-oxidant and anti-inflammatory effects [1-7]. Traditionally melatonin is administered orally, but due to extensive first pass metabolism melatonin displays a poor and variable bioavailability [8-11]. Furthermore, studies have described a variable absorption of oral melatonin from the gastrointestinal tract [24]. These pharmacokinetic properties may advocate for alternative administration routes of melatonin.

\section{Intranasal administration}

The included studies documented a rapid $\mathrm{T}_{\max }$ of intranasal administration, ranging between 2.5 and 7.8 min depending on the subjects (animals and humans) and formulation [14-17]. These properties suggest that melatonin is easily transferred across the nasal mucosa. A study in rabbits demonstrated $T_{1 / 2}$ values similar to that of the I.V. administration [16]. Bioavailability was investigated in 2 studies employing rabbits [16,17], and ranged between 55 and $94 \%$. These findings indicate that intranasal administration of melatonin provides a significantly higher bioavailability compared to oral melatonin, which is estimated to approximately $15 \%[8,11]$. No studies in humans, investigating bioavailability, have been performed yet, but the rapid absorption phase and high bioavailability in rabbits makes I.N. administration of melatonin relevant in future clinical research, where a systemic effect is wanted. Intranasal administration of melatonin could prove relevant in e.g., treatment of jet-lag, due to the ease of administration as a nasal spray.

\section{Transdermal administration}

The 4 human studies that investigated transdermal administration of melatonin and documented $\mathrm{T}_{\max }$ ranging between 1 and 18.12 h, with substantial inter individual variation [18-21]. Due to the slow transdermal absorption of the melatonin, just one study was able to estimate $T_{1 / 2}$. Only 2 subjects were included and 
$T_{1 / 2}$ varied extensively between them [19]. Furthermore, one study demonstrated a deposition of melatonin in the skin, causing the plasma concentration of melatonin to rise after removal of the melatonin-patch [20]. Transdermal administration provides an easy administration route, but so far only a limited number of low volume studies have been performed in humans. Also, the studies used different formulations of melatonin for transdermal delivery, which might contribute to the varying results [18-21]. Development of other formulations should be applied in order to increase absorption rates and provide stable plasma concentrations, if systemic effects of melatonin are intended. If a local effect of melatonin is intended, transdermal administration of melatonin could potentially be used to protect the skin from UV-radiation [25].

\section{Oral transmucosal administration}

2 human studies investigated oral transmucosal administration, employing a buccal patch [20] and an oral sublingual spray, respectively [22]. Very variable $\mathrm{T}_{\max }$ values were demonstrated, documenting an extended absorption phase in the study with the buccal patch [20]. The study administering the sublingual spray also found significantly higher $C_{\max }$ values compared to conventional oral administration [22]. The results may be due to lack of first-pass metabolism in oral transmucosal administration. Hence, these collective pharmacokinetic advantages are comparable to I.N. administration. Oral transmucosal administration of melatonin could possibly replace conventional oral administration to improve sleep quality, due to the reliable $T_{\max }$, making it easier to predict the time from administration to effect of the melatonin.

\section{Subcutaneous injection}

One study in sheep investigated subcutaneous injection, and demonstrated a lower $\mathrm{T}_{\max }$ compared to oral administration [23]. Currently, no studies in humans have investigated subcutaneous injections. From a clinical point of view subcutaneous injections may seem unreasonable, due to the variable and low absorption in subcutaneous tissues.

\section{Strenghts and limitations}

This was the first systematic review of the alternative administration routes of melatonin. The review was performed in accordance to the PRISMA and PRISMA-P guidelines $[12,13]$. We documented that only a limited number of studies have investigated this subject.

Our review only included 10 studies, and the studies varied extensively in design, subject species, the number of subjects, measuring periods, methods of analyses, dose of melatonin, formulations of melatonin, and the pharmacokinetic outcomes reported.

\section{Conclusions}

$\nabla$

This review demonstrated that only a limited number of studies have investigated alternative administration routes of melatonin. The studies varied in both subjects and pharmacokinetic outcomes. I.N. administration demonstrated a higher bioavailability and $\mathrm{T}_{\max }$ compared to oral melatonin. Transdermal administration of melatonin might be used optimally in a local application, rather than a systemic application, due to slow absorption of melatonin, and deposition of melatonin in the skin. The oral transmucosal route demonstrated higher $C_{\max }$ values with similar $\mathrm{T}_{\max }$ values compared to oral melatonin. Oral transmucosal administration may potentially be a clinically relevant administration route if a systemic effect is wanted. Currently there are no studies investigating subcutaneous injection of melatonin in humans, and studies in animals did not document any advantages compared to other administration routes.

\section{Conflict of Interest}

$\nabla$

The authors have no affiliations with or involvement in any organization or entity with any financial interest or non-financial interest in the subject matter or materials discussed in this review.

\section{References}

1 Nir I. Melatonin for the treatment of disorders in circadian rhythm and sleep: could it form a basis for medication? Receptors Channels 2003; 9: 379-385

2 Garfinkel D, Laudon M, Nof D et al. Improvement of sleep quality in elderly people by controlled-release melatonin. Lancet 1995; 346: 541-544

3 Petrie K, Dawson AG, Thompson L et al. A double-blind trial of melatonin as a treatment for jet lag in international cabin crew. Biol Psychiatry 1993; 33: 526-530

4 Borazan H, Tuncer S, Yalcin N et al. Effects of preoperative oral melatonin medication on postoperative analgesia, sleep quality, and sedation in patients undergoing elective prostatectomy: a randomized clinical trial. J Anesth 2010; 24: 155-160

5 Caumo W, Torres F, Moreira NL Jr et al. The clinical impact of preoperative melatonin on postoperative outcomes in patients undergoing abdominal hysterectomy. Anesth Analg 2007; 105: 1263-1271 table of contents

6 Gitto E, Romeo C, Reiter RJ et al. Melatonin reduces oxidative stress in surgical neonates. J Pediatr Surg 2004; 39: 184-189 discussion-9

7 Kucukakin B, Lykkesfeldt J, Nielsen HJ et al. Utility of melatonin to treat surgical stress after major vascular surgery - a safety study. J Pineal Res 2008; 44: 426-431

8 DeMuro RL, Nafziger AN, Blask DE et al. The absolute bioavailability of oral melatonin. J Clin Pharmacol 2000; 40: 781-784

9 Lane $E A$, Moss $H B$. Pharmacokinetics of melatonin in man: first pass hepatic metabolism. J Clin Endocrinol Metab 1985; 61: 1214-1216

$10 \mathrm{Di}$ WL, Kadva A, Johnston A et al. Variable bioavailability of oral melatonin. N Engl J Med 1997; 336: 1028-1029

11 Harpsoe NG, Andersen LP, Gogenur I et al. Clinical pharmacokinetics of melatonin: a systematic review. Eur J Clin Pharmacol 2015; 71: 901-909

12 Moher D, Liberati A, Tetzlaff J et al. Preferred reporting items for systematic reviews and meta-analyses: the PRISMA statement. Int J Surg 2010; 8: 336-341

13 Moher D, Shamseer L, Clarke $M$ et al. Preferred reporting items for systematic review and meta-analysis protocols (PRISMA-P) 2015 statement. Syst Rev 2015; $4: 1$

14 van den Berg MP, Merkus P, Romeijn SG et al. Uptake of melatonin into the cerebrospinal fluid after nasal and intravenous delivery: studies in rats and comparison with a human study. Pharm Res 2004; 21: 799-802

15 Merkus P, Guchelaar HJ, Bosch DA et al. Direct access of drugs to the human brain after intranasal drug administration? Neurology 2003; 60: $1669-1671$

16 Bechgaard E, Lindhardt K, Martinsen L. Intranasal absorption of melatonin in vivo bioavailability study. Int J Pharm 1999; 182: 1-5

17 Mao S, Chen J, Wei Z et al. Intranasal administration of melatonin starch microspheres. Int J Pharm 2004; 272: 37-43

18 Aeschbach D, Lockyer BJ, Dijk DJ et al. Use of transdermal melatonin delivery to improve sleep maintenance during daytime. Clin Pharmacol Ther 2009; 86: 378-382

19 Priano L, Esposti D, Esposti $R$ et al. Solid lipid nanoparticles incorporating melatonin as new model for sustained oral and transdermal delivery systems. J Nanosci Nanotechnol 2007; 7: 3596-3601 
20 Benes L, Claustrat B, Horriere F et al. Transmucosal, oral controlledrelease, and transdermal drug administration in human subjects: a crossover study with melatonin. J Pharm Sci 1997; 86: 1115-1119

21 Bangha E, Lauth D, Kistler GS et al. Daytime serum levels of melatonin after topical application onto the human skin. Skin Pharmacol 1997; 10: 298-302

22 Bartoli AN, De Gregori S, Molinaro M et al. Bioavailability of a new oral spray melatonin emulsion compared with a standard oral formulation in healthy volunteers. Journal of Bioequivalence and Bioavailability 2012; 4: 96-99
23 Kennaway DJ, Seamark RF. Circulating levels of melatonin following its oral administration or subcutaneous injection in sheep and goats. Aust J Biol Sci 1980; 33: 349-353

24 Fourtillan JB, Brisson AM, Gobin P et al. Bioavailability of melatonin in humans after day-time administration of $\mathrm{D}(7)$ melatonin. Biopharm Drug Dispos 2000; 21: 15-22

25 Scheuer C, Pommergaard HC, Rosenberg J et al. Melatonin's protective effect against UV radiation: a systematic review of clinical and experimental studies. Photodermatol Photoimmunol Photomed 2014; 30: $180-188$ 